\title{
Two Approximations of Solutions of Hamilton-Jacobi Equations*
}

\author{
By M. G. Crandall** and P. L. Lions
}

\begin{abstract}
Equations of Hamilton-Jacobi type arise in many areas of application, including the calculus of variations, control theory and differential games. The associated initial-value problems almost never have global-time classical solutions, and one must deal with suitable generalized solutions. The correct class of generalized solutions has only recently been established by the authors. This article establishes the convergence of a class of difference approximations to these solutions by obtaining explicit error estimates. Analogous results are proved by similar means for the method of vanishing viscosity.
\end{abstract}

Introduction. The main results of this paper concern the approximation of solutions of the Cauchy problem for first-order partial differential equations of Hamilton-Jacobi type. Most of the presentation here will be in the context of problems of the form

$$
\begin{cases}\partial u / \partial t+H(D u)=0 & \text { in } \mathbf{R}^{N} \times(0, \infty), \\ u(x, 0)=u_{0}(x) & \text { in } \mathbf{R}^{N},\end{cases}
$$

where $H \in C\left(\mathbf{R}^{N}\right)$ (the continuous functions on $\left.\mathbf{R}^{N}\right), u_{0} \in \operatorname{BUC}\left(\mathbf{R}^{N}\right.$ ) (the bounded and uniformly continuous functions on $\left.\mathbf{R}^{N}\right)$, and $D u=\left(u_{x_{1}}, \ldots, u_{x_{N}}\right)$ is the spatial gradient of $u$. The problem (IVP) is technically simpler than the "general case" in which the Hamiltonian $H$ may depend on $x, t$ and $u$ as well as $D u$, and we prefer to keep the ideas clear and constants simple by dealing primarily with (IVP). (See the comments in Section 4 regarding more general equations.) Two sorts of approximations of (IVP) will be considered here-finite difference schemes and the method of vanishing viscosity. Before describing these approximations, we briefly review some basic facts concerning (IVP).

Analysis by the method of chacteristics shows that if $H$ and $u_{0}$ are smooth and $u_{0}$ is compactly supported, then (IVP) will typically have a unique $C^{2}$ solution $u$ on some maximal time interval $0 \leqslant t<T$ for which $\lim _{t \uparrow T} u(x, t)$ exists uniformly, but this limiting function is not continuously differentiable. Thus $D u$ "becomes discontinuous" at $t=T$ (or "shocks form"). If one insists upon a solution of (IVP) which is defined for all $t>0$, it is therefore necessary to deal with functions which are not

Received September 28, 1982.

1980 Mathematics Subject Classification. Primary 65M15, 65M10.

Key words and phrases. Hamilton-Jacobi equations, difference approximations, error estimates, method of vanishing viscosity.

*Sponsored by the United States Army under Contract No. DAAG29-80-C-0041.

**Supported in part by the National Science Foundation under Grant No. MCS-8002946. 
smooth. On the other hand, it is relatively easy, in the above circumstances, to produce Lipschitz continuous functions $u$ on $\mathbf{R}^{N} \times[0, \infty)$ which satisfy (IVP) if the equation is understood in the "almost everywhere" sense. However, "generalized" solutions in this sense are not unique.

Recently, a way of identifying a uniquely existing solution for a class of problems which include (IVP) as a special case was given by the authors in [2], [3] (see also [1]). The relevant solutions of scalar nonlinear first order equations are called "viscosity solutions", and they are known to be the solutions of primary interest in many areas of application (e.g., optimization, control theory, differential games, etc.). See, e.g., [6], [10], [11]. The term "viscosity solutions" refers to the fact that all solutions obtained via the method of vanishing viscosity are in this class. The main properties of viscosity solutions relevant for the current work are recalled, in the context of (IVP), in Section 1.

In this paper we will approximate the viscosity solution of (IVP) by solutions of the general class of finite difference schemes introduced below. Indeed, explicit error estimates are given relating the viscosity solution of (IVP) and the solutions of these finite difference approximations. We also show, under suitable hypotheses, that if $\varepsilon>0, u^{\varepsilon}$ is the solution of the problem

$$
\begin{cases}\partial u^{\varepsilon} / \partial t+H\left(D u^{\varepsilon}\right)-\varepsilon \Delta u^{\varepsilon}=0 & \text { in } \mathbf{R}^{N} \times(0, \infty), \\ u^{\varepsilon}(x, 0)=u_{0}(x) & \text { in } \mathbf{R}^{N},\end{cases}
$$

and $u$ is the viscosity solution of (IVP), then $\left|u^{\varepsilon}(x, t)-u(x, t)\right| \leqslant c \sqrt{\varepsilon}$ for $x \in \mathbf{R}^{N}$ and $t>0$. This is done in Section 5. Estimates like this have also been obtained in W. H. Fleming [7] and P. L. Lions [10] by indirect arguments involving stochastic differential games.

We now describe the class of difference approximations to be considered here. For notational simplicity only, we will assume that $N=2$ in most of the presentation. The corresponding definitions and results for general $N$ will be clear from this special case, and we will not explicitly formulate them. A generic point in $\mathbf{R}^{2}$ will be denoted by $(x, y)$, and we will write $D u=\left(u_{x}, u_{y}\right)$. Given mesh sizes $\Delta x, \Delta y, \Delta t>0$, the value of our numerical approximation at $\left(x_{j}, y_{k}, t_{n}\right)=(j \Delta x, k \Delta y, n \Delta t)$ $(j, k, n \in \mathbf{Z})$ will be denoted by $U_{j, k}^{n}$. Capital letters $U, V, \ldots$ will denote functions on the $x, y$ lattice $\Delta=\left\{\left(x_{j}, y_{k}\right): j, k \in \mathbf{Z}\right\}$ and their values at $\left(x_{j}, y_{k}\right)$ will be written $U_{j, k}, V_{j, k}, \ldots$ Thus $U^{n}$ represents the state of our numerical approximation at the time level $n \Delta t$, and it is a function on $\Delta$ with values $U_{j, k}^{n}$. The notations $\lambda^{x}=\Delta t / \Delta x, \lambda^{y}=\Delta t / \Delta y, \Delta_{+}^{x} U_{j, k}=U_{j+1, k}-U_{j, k}$, and $\Delta_{+}^{y} U_{j, k}=U_{j, k+1}-U_{j, k}$ will be used.

The discrete approximations of (IVP) of interest here are explicit marching schemes of the form

$$
U_{j, k}^{n+1}=G\left(U_{j-p, k-r}^{n}, \ldots, U_{j+q+1, k+s+1}^{n}\right),
$$

where $p, q, r, s$ are fixed nonnegative integers and $G$ is a function of $(p+q+2)$ $\cdot(r+s+2)$ variables. (At this stage we are ignoring the dependence of $G$ on $\Delta x, \Delta y$ and $\Delta t$.) To simplify notation, (1) will also be written as

$$
U^{n+1}=\vec{G}\left(U^{n}\right) \text {. }
$$


We say that (1) has "differenced form" if there exists a function $g$ such that

$$
\begin{aligned}
G\left(U_{j-p, k-r}, \ldots, U_{j+q+1, k+s+1}\right)= & U_{j_{1}, k} \\
& -\Delta \operatorname{tg}\left(\frac{\Delta_{+}^{x}}{\Delta x} U_{j-p, k-r}, \ldots, \frac{\Delta_{+}^{x}}{\Delta x} U_{j+q, k+s+1} ;\right. \\
& \left.\frac{\Delta_{+}^{y}}{\Delta y} U_{j-p, k-r}, \ldots, \frac{\Delta_{+}^{y}}{\Delta y} U_{j+q+1, k+s}\right) .
\end{aligned}
$$

In order that the scheme (1), (3) be consistent with the equation $u_{t}+H\left(u_{x}, u_{y}\right)=0$ occurring in (IVP), we must have

$$
g(a, \ldots, a ; b, \ldots, b)=H(a, b) \text { for } a, b \in \mathbf{R}
$$

When (3) holds we call $g$ the numerical Hamiltonian of the scheme. Finally, we will say that (2) (or (3)) is monotone on $[-R, R]$ if $G\left(U_{j-p, k-r}, \ldots, U_{j+q+1, k+s+1}\right)$ is a nondecreasing function of each argument as long as $\left|\Delta_{+}^{x} U_{l, m}\right| / \Delta x,\left|\Delta_{+}^{y} U_{l^{\prime}, m^{\prime}}\right| / \Delta y \leqslant R$ for $j-p \leqslant l \leqslant j+q, k-r \leqslant m \leqslant k+s+1, j-p \leqslant l^{\prime} \leqslant j+q+1, k-r \leqslant m^{\prime}$ $\leqslant k+s$. Roughly speaking, $R$ will be an a priori bound on $\left|u_{x}\right|,\left|u_{y}\right|$ for the solution of (IVP).

Our main result is

TheOREM 1. Let $H: \mathbf{R}^{2} \rightarrow \mathbf{R}$ be continuous and $u_{0}$ be bounded and Lipschitz continuous on $\mathbf{R}^{2}$ with $L$ as a Lipschitz constant. For $\lambda^{x}, \lambda^{y}>0$ and fixed, let the scheme (2) have differenced form, be monotone on $[-(L+1), L+1]$ and be consistent with (IVP). Assume the numerical Hamiltonian $g$ is locally Lipschitz continuous. Define $U^{0}$ by $U_{j, k}^{0}=u_{0}\left(x_{j}, y_{k}\right)$ and then $U^{n}, n=1,2, \ldots$, by (2). Let $u$ be the viscosity solution of (IVP). Then there is a constant $c$ depending only on $\sup \left|u_{0}\right|, L, g$ and $N \Delta t$ such that

$$
\left|U_{j, k}^{n}-u\left(x_{j}, y_{k}, n \Delta t\right)\right| \leqslant c(\sqrt{\Delta t})
$$

for $0 \leqslant n \leqslant N$ and all $j, k$.

The body of the paper is structured in the following way: Section 1 is devoted to a review of the properties of viscosity solutions as needed herein. Examples of difference schemes satisfying the assumptions of Theorem 1 are presented in Section 2. Theorem 1 is proved in Sections 3 and 4, with Section 3 consisting of preparatory lemmas on the mapping $U \rightarrow \vec{G}(U)$, while Section 4 contains the proof of (5). Section 4 concludes with remarks on variations of Theorem 1. The approximation of (IVP) by (IVP) $)_{\varepsilon}$ is treated in Section 5.

We bring this long introduction to a close with some remarks: First of all, some convergence results are given in S. N. Kružkov [8] for convex Hamiltonians $H$, using some estimates available only in this special case. Next (see, e.g., P. L. Lions [10, Chapter 16]) problems like (IVP) are closely related to hyperbolic systems satisfied 
by $D u$. If $N=1$, this relation is quite simple, since if $u$ solves (IVP), then $v=u_{x}$ solves a scalar conservation law:

$$
\begin{cases}\frac{\partial v}{\partial t}+\frac{\partial}{\partial x}(H(v))=0 & \text { in } \mathbf{R} \times(0, \infty), \\ v(x, 0)=v_{0}(x)=\frac{d u_{0}}{d x}(x) & \text { in } \mathbf{R} .\end{cases}
$$

In this case the schemes presented here are related to those studied by M. G. Crandall and A. Majda [4], and N. N. Kuznetsov [9] via the corresponding substitution:

$$
V_{k}^{n}=\frac{1}{\Delta x} \Delta_{+} U_{k}^{n}=\frac{U_{k+1}^{n}-U_{k}^{n}}{\Delta x}
$$

We refer the reader interested in other aspects of Hamilton-Jacobi equations to M. G. Crandall and P. L. Lions [2], P. L. Lions [10], and M. G. Crandall, L. C. Evans and P. L. Lions [1]. Finally we remark that, in an ongoing investigation, P. Souganidis [11] has formulated general approximation results which appear to apply alike to dimensional splitting, max-min representations, approximation by $(\text { IVP) })_{\varepsilon}$ and numerical schemes. His arguments are related to those given herein.

1. Viscosity Solutions of (IVP). As recalled in the Introduction, one cannot solve (IVP) in a classical way on $\mathbf{R}^{N} \times(0, \infty)$ in general, while Lipschitz continuous "generalized solutions" in the almost everywhere sense exist under mild assumptions but are not unique (examples are given, for instance, in [2]). The resolution of these difficulties is given in [2], the results of which imply, in a roundabout way, the theorem stated below. This theorem is proved directly in [1]. (We use $x$ to denote points in $\mathbf{R}^{N}$ below.)

Theorem (Existence ANd UniQueness). Let $H \in C\left(\mathbf{R}^{N}\right), u_{0} \in \mathrm{BUC}\left(\mathbf{R}^{N}\right)$. Then there is exactly one function $u \in \mathrm{BUC}\left(\mathbf{R}^{N} \times[0, T]\right)$ for all $T>0$ such that $u(x, 0)=$ $u_{0}(x)$, and for every $\phi \in C^{1}\left(\mathbf{R}^{N} \times(0, \infty)\right)$ and $T>0$ :

$$
\left\{\begin{array}{c}
\text { If }\left(x_{0}, t_{0}\right) \text { is a local maximum point of } u-\phi \text { on } \mathbf{R}^{N} \times(0, T], \text { then } \\
\frac{\partial \phi}{\partial t}\left(x_{0}, t_{0}\right)+H\left(D \phi\left(x_{0}, t_{0}\right)\right) \leqslant 0
\end{array}\right.
$$

and

$$
\left\{\begin{array}{c}
\text { If }\left(x_{0}, t_{0}\right) \text { is a local minimum point of } u-\phi \text { on } \mathbf{R}^{N} \times(0, T], \text { then } \\
\frac{\partial \phi}{\partial t}\left(x_{0}, t_{0}\right)+H\left(D \phi\left(x_{0}, t_{0}\right)\right) \geqslant 0 .
\end{array}\right.
$$

The function $u$ whose existence and uniqueness is asserted by the theorem is called the viscosity solution of (IVP). A continuous function $u$ on $\mathbf{R}^{N} \times[0, T]$ which satisfies (1.1), (1.2) is called a viscosity solution of the equation $u_{t}+H(D u)=0$ on $\mathbf{R}^{N} \times$ $[0, T]$. See [1], [2] for the appropriate notions for more general equations. There are also useful equivalent ways to formulate the notion of viscosity solutions [1], [2]. Among the desirable properties of the notion of viscosity solutions is its consistency 
with the classical concept. That is, if $u$ is a classical (i.e., $C^{1}$ ) solution of $u_{t}+H(D u)$ $=0$, then it is a viscosity solution, and if $u$ is a viscosity solution, then $u_{t}\left(x_{0}, t_{0}\right)+$ $H\left(D u\left(x_{0}, t_{0}\right)\right)=0$ at any point $\left(x_{0}, t_{0}\right)$ where $u$ is differentiable.

The other information we want to recall consists of various estimates on the behavior of solutions of (IVP). To record these, for each $t \geqslant 0$ let $S(t)$ : $\mathrm{BUC}\left(\mathbf{R}^{N}\right) \rightarrow$ $\operatorname{BUC}\left(\mathbf{R}^{N}\right)$ be the time $t$ map associated with (IVP). That is, $S(t) u_{0}(x)=u(x, t)$ where $u$ is the viscosity solution of (IVP). We also put $\|f\|=\sup _{\mathbf{R}^{N}}|f(x)|$ and $f^{+}=\max (f, 0)$. The next result follows from [2], see also [1].

Proposition 1.1. Let $H \in C\left(\mathbf{R}^{N}\right)$ and $S(t)$ be as above; $u_{0}, v_{0} \in \mathrm{BUC}\left(\mathbf{R}^{N}\right)$, and $t \geqslant 0$. Then

(i) $\left\|\left(S(t) u_{0}-S(t) v_{0}\right)^{+}\right\| \leqslant\left\|\left(u_{0}-v_{0}\right)^{+}\right\|$.

(ii) $\left\|S(t) u_{0}-S(t) v_{0}\right\| \leqslant\left\|u_{0}-v_{0}\right\|$.

(iii) $\inf _{\mathbf{R}^{N}} u_{0} \leqslant t H(0)+S(t) u_{0} \leqslant \sup _{\mathbf{R}^{N}} u_{0}$.

(iv) $\left|S(t) u_{0}(x+y)-S(t) u_{0}(x)\right| \leqslant \sup _{z \in \mathbf{R}^{N}}\left|u_{0}(z+y)-u_{0}(z)\right|$, for $y \in \mathbf{R}^{N}$.

(v) If $L$ is a Lipschitz constant for $u_{0}$, then it is also a Lipschitz constant for

$$
S(t) u_{0} \text { and }\left\|S(t) u_{0}-S(\tau) u_{0}\right\| \leqslant|t-\tau| \sup _{\mathbf{R}^{N}}\{|H(p)|:|p| \leqslant L\} .
$$

The key point here is (i). The estimate (i) implies (ii) upon using (i) with $u_{0}$ and $v_{0}$ interchanged. Clearly (i) also implies $S(t) u_{0} \geqslant S(t) v_{0}$ if $u_{0} \geqslant v_{0}$, which in turn implies (iii) since $v=c-t H(0)$ is a classical (and so the viscosity) solution of (IVP) with the constant initial datum $c$. Choosing $v_{0}=\sup u_{0}$ or inf $u_{0}$ and using the orderpreserving property yields (iii). Next (iv) follows from (ii) because $S(t) u_{0}(\cdot+y)$ is the solution of (IVP) for the initial datum $u_{0}(\cdot+y)$. Since (iv) shows that a modulus of continuity for $u_{0}$ is also a modulus for $S(t) u_{0}(\cdot)$, the first assertion of (v) is clear. The Lipschitz property in the time is easily deduced from the equation $u_{t}+H(D u)$ $=0$ in the viscosity sense (see [2]) or in other ways.

2. Examples. We begin with $N=1$ and write (IVP) in the form

$$
\begin{cases}u_{t}+H\left(u_{x}\right)=0, & \text { for } t>0, x \in \mathbf{R} \\ u(x, 0)=u_{0}(x), & \text { for } x \in \mathbf{R}\end{cases}
$$

in this case. As the first example, we consider the scheme

$$
U_{j}^{n+1}=U_{j}^{n}-\Delta t\left\{H\left(\frac{U_{j+1}^{n}-U_{j-1}^{n}}{2 \Delta x}\right)-\frac{\theta}{\lambda^{x}} \frac{U_{j+1}^{n}+U_{j-1}^{n}-2 U_{j}^{n}}{\Delta x}\right\},
$$

where $\theta>0$ is given. The relation (2.2) may be rewritten as

$$
U_{j}^{n+1}=U_{j}^{n}-\Delta t\left\{H\left(\frac{\Delta_{+}^{x} U_{j}^{n}+\Delta_{+}^{x} U_{j-1}^{n}}{2 \Delta x}\right)-\frac{\theta}{\lambda^{x}}\left(\frac{\Delta_{+}^{x} U_{j}^{n}-\Delta_{+}^{x} U_{j-1}^{n}}{\Delta x}\right)\right\},
$$

making the differenced form clear. The numerical Hamiltonian is given by

$$
g(\alpha, \beta)=H((\alpha+\beta) / 2)-(\beta-\alpha) \theta / \lambda^{x} \quad \text { for } \alpha, \beta \in \mathbf{R} .
$$

Clearly $g(\alpha, \alpha)=H(\alpha)$ and so (2.2) is consistent. The scheme (2.2) is monotone on $[-R, R]$ if $1-2 \theta \geqslant 0$ (monotonicity in $U_{j}^{n}$ ), and $\theta-\lambda^{x}\left|H^{\prime}(\alpha)\right| / 2 \geqslant 0$ for $|\alpha| \leqslant R$ (monotonicity in $U_{j+1}^{n}, U_{j-1}^{n}$ ). These two relations are achieved by first choosing $0<\theta<1 / 2$ and then $\lambda^{x}$ sufficiently small. This scheme is analogous to the Lax-Friedrichs scheme for conservation laws; see [4]. 
In a similar way, the schemes

$$
U_{j}^{n+1}=U_{j}^{n}-\Delta t H\left(\frac{U_{j+1}^{n}-U_{j}^{n}}{\Delta x}\right),
$$

or

$$
U_{j}^{n+1}=U_{j}^{n}-\Delta t H\left(\frac{U_{j}^{n}-U_{j-1}^{n}}{\Delta x}\right),
$$

have the desired properties if $H$ is nonincreasing (for (2.3)), if $H$ is nondecreasing (for (2.4)), and $1 \geqslant \lambda^{x}\left|H^{\prime}(\alpha)\right|$ for $|\alpha| \leqslant R$. Those are simple "upwind" schemes. Next, let $\alpha_{0} \in R$, and assume $H^{\prime}(\alpha)\left(\alpha-\alpha_{0}\right) \geqslant 0$, so $H^{\prime}$ changes sign at $\alpha=\alpha_{0}$. Set $\theta(s)=1$ if $s \leqslant \alpha_{0}, \theta(s)=0$ if $s>\alpha_{0}$. We consider the scheme

$$
\begin{aligned}
U_{j}^{n+1}= & U_{j}^{n}-\Delta t\left\{\theta\left(\frac{U_{j+1}^{n}-U_{j}^{n}}{\Delta x}\right)\left\{H\left(\frac{U_{j+1}^{n}-U_{j}^{n}}{\Delta x}\right)-H\left(\alpha_{0}\right)\right\}\right. \\
& \left.+\left(1-\theta\left(\frac{U_{j}^{n}-U_{j-1}^{n}}{\Delta x}\right)\right)\left\{H\left(\frac{U_{j}^{n}-U_{j-1}^{n}}{\Delta x}\right)-H\left(\alpha_{0}\right)\right\}+H\left(\alpha_{0}\right)\right\} .
\end{aligned}
$$

The numerical Hamiltonian is now

$$
g(\alpha, \beta)=\theta(\beta)\left\{H(\beta)-H\left(\alpha_{0}\right)\right\}+(1-\theta(\alpha))\left(H(\alpha)-H\left(\alpha_{0}\right)\right)+H\left(\alpha_{0}\right),
$$

and thus (2.5) is consistent. Remarking that $g(\alpha, \beta)$ may be written as $g(\alpha, \beta)=$ $H\left(\beta \wedge \alpha_{0}\right)+H\left(\alpha \vee \alpha_{0}\right)-H\left(\alpha_{0}\right)$, where " $\wedge$ " and " $\vee$ " denote "maximum" and "minimum", it is clear that $g$ is locally Lipschitz if $H$ is locally Lipschitz. Finally, one checks that (2.5) is monotone on $[-R,+R]$ if $1-\lambda^{x}\left|H^{\prime}(\alpha)\right| \geqslant 0$ for $|\alpha| \leqslant R$.

In fact, all the above examples are merely adaptations to Hamilton-Jacobi equations of well-known schemes for conservation laws via the remarks in the Introduction. As explained in, e.g., M. G. Crandall and A. Majda [4], there is a class of schemes for the conservation law $v_{t}+(H(v))_{x}=0$, called "monotone, in conservation form" with the following structure: $V_{j}^{n+1}=V_{j}^{n}-\lambda^{x} \Delta_{+}^{x} g\left(V_{j-p}^{n}, \ldots, V_{j+q}^{n}\right)$ - now the function $g$ is called the "numerical flux". Consistency then means, as before, $g(\alpha, \ldots, \alpha)=H(\alpha)$ and monotonicity means that the map $\left(V^{n} \mapsto V_{j}^{n+1}\right)$ is a nondecreasing function of each $V_{l}^{n}$. Then we may write the corresponding scheme for the approximation of (IVP),

$$
U_{j}^{n+1}=U_{j}^{n}-\Delta \operatorname{tg}\left(\frac{\Delta_{+}^{x} U_{j-p}^{n}}{\Delta x}, \ldots, \frac{\Delta_{+}^{x} U_{j+q}^{n}}{\Delta x}\right),
$$

which is in differenced form and consistent.

Next, if $N>1$, the relation between (IVP) and conservation laws disappears. For $N=2$ (to simplify, as always) we mention the analogue of the Lax-Friedrichs scheme, i.e.

$$
\begin{array}{rl}
U_{j, k}^{n+1}=U_{j, k}^{n}-\Delta t & H\left(\frac{U_{j+1, k}^{n}-U_{j-1, k}^{n}}{2 \Delta x}, \frac{U_{j, k+1}^{n}-U_{j, k-1}^{n}}{2 \Delta y}\right) \\
& -\frac{\theta}{\lambda^{x}}\left(\frac{U_{j+1, k}^{n}+U_{j-1, k}^{n}-2 U_{j, k}^{n}}{\Delta x}\right) \\
& \left.-\frac{\theta}{\lambda^{y}}\left(\frac{U_{j, k+1}^{n}+U_{j, k-1}^{n}-2 U_{j, k}^{n}}{\Delta y}\right)\right\},
\end{array}
$$


which is consistent, in differenced form. It is also monotone on $[-R, R]$ provided that $0<\theta<1 / 4, \theta-\lambda^{x}\left|H_{1}^{\prime}(\alpha, \beta)\right| \geqslant 0$, and $\theta-\lambda^{y}\left|H_{2}^{\prime}(\alpha, \beta)\right| \geqslant 0$ for $|\alpha|,|\beta| \leqslant R$, where $H_{i}^{\prime}$ denotes the derivative of $H$ in its $i$ th argument.

As a last example, let

$$
\begin{aligned}
V_{j, k}^{n+1}= & G_{1}\left(V_{j-p, k-r}^{n}, \ldots, V_{j+q+1, k+s+1}^{n}\right) \\
= & V_{j, k}^{n}-\Delta \operatorname{tg} 1\left(\frac{\Delta_{+}^{x} V_{j-p, k-r}^{n}}{\Delta x}, \ldots, \frac{\Delta_{+}^{x} V_{j+q, k+s+1}^{n}}{\Delta x} ;\right. \\
& \left.\frac{\Delta_{+}^{y} V_{j-1, k-r}^{n}}{\Delta y}, \ldots, \frac{\Delta_{+}^{y} V_{j+q+1, k+s}^{n}}{\Delta y}\right)
\end{aligned}
$$

and

$$
\begin{aligned}
W_{j, k}^{n+1}= & G_{2}\left(W_{j-p, k-r}^{n}, \ldots, W_{j+q+1, k+s+1}^{n}\right) \\
= & W_{j, k}^{n}-\Delta \operatorname{tg}_{2}\left(\frac{\Delta_{+}^{x} W_{j-p, k-r}^{n}}{\Delta x}, \ldots, \frac{\Delta_{x}^{+} W_{j+q, k+s+1}^{n}}{\Delta x} ;\right. \\
& \left.\frac{\Delta_{+}^{y} W_{j-p, k-r}^{n}}{\Delta y}, \ldots, \frac{\Delta_{+}^{y} W_{j+q+1, k+s}^{n}}{\Delta y}\right)
\end{aligned}
$$

be differenced form, monotone on $[-R, R]$ schemes consistent with $v_{t}+$ $\alpha^{-1} H_{1}\left(v_{x}, v_{y}\right)=0$ and $w_{t}+(1-\alpha)^{-1} H_{2}\left(w_{x}, w_{y}\right)=0$, respectively, where $\alpha \in(0,1)$. Then the scheme

$$
\begin{aligned}
U_{j, k}^{n+1}= & \alpha G_{1}\left(U_{j-p, k-r}^{n}, \ldots, U_{j+q+1, k+s+1}^{n}\right) \\
& +(1-\alpha) G_{2}\left(U_{j-p, k-r}^{n}, \ldots, U_{j+q+1, k+s+1}^{n}\right)
\end{aligned}
$$

has differenced form, is monotone on $[-R, R]$ and is consistent with: $\partial u / \partial t+$ $H_{1}\left(u_{x}, u_{y}\right)+H_{2}\left(u_{x}, u_{y}\right)=0$. E.g., we could build schemes in this way for

$$
\frac{\partial u}{\partial t}+H_{1}\left(\frac{\partial u}{\partial x}\right)+H_{2}\left(\frac{\partial u}{\partial y}\right)=0
$$

from schemes for

$$
\frac{\partial u}{\partial t}+H_{1}\left(\frac{\partial u}{\partial x}\right)=0 \text { and } \frac{\partial w}{\partial t}+H_{2}\left(\frac{\partial w}{\partial y}\right)=0 .
$$

3. Stability Properties of the Schemes. The notation in this section assumes two space dimensions, but everything herein easily generalizes to arbitrary dimensions. By capital letters $U, V$, etc., we denote bounded bi-infinite double sequences $\left\{U_{j, k}\right\}_{(j, k) \in \mathbf{Z}^{2}}$, i.e. bounded functions on $\mathbf{Z}^{2}$, the set of all such being $l^{\infty}\left(\mathbf{Z}^{2}\right)$, which we equip with the norm

$$
\|U\|_{\infty}=\sup _{j, k \in \mathbf{Z}}\left|U_{j, k}\right|
$$

Let $\vec{G}$ be the self-map of $l^{\infty}\left(\mathbf{Z}^{2}\right)$ defined by (3), i.e.

$$
\begin{aligned}
\vec{G}(U)_{j, k}=U_{j, k}-\Delta t g\left(\frac{\Delta_{+}^{x} U_{j-p, k-r}}{\Delta x}\right. & , \ldots, \frac{\Delta_{+}^{y} U_{j+q, k+s+1}}{\Delta x} ; \\
& \left.\frac{\Delta_{+}^{y} U_{j-p, k-r}}{\Delta y}, \ldots, \frac{\Delta_{+}^{y} U_{j+q+1, k+s}}{\Delta y}\right) .
\end{aligned}
$$


We now investigate the properties of $\vec{G}$. By definition (2), (3) is monotone on $[-R, R]$ if the restriction of $\vec{G}$ to

$$
C=\left\{U \in l^{\infty}\left(\mathbf{Z}^{2}\right):\left|\Delta_{+}^{x} U_{l, m}\right| \leqslant R \Delta x,\left|\Delta_{+}^{y} U_{l, m}\right| \leqslant R \Delta y, \text { for } l, m \in \mathbf{Z}\right\}
$$

preserves the natural ordering of $l^{\infty}\left(\mathbf{Z}^{2}\right)$. We identify $\lambda \in \mathbf{R}$ with the constant function $\lambda$ on $\mathbf{Z}^{2}$. From the form of $G$, it is clear that

$$
\vec{G}(U+\lambda)=\vec{G}(U)+\lambda \text { for all } U \in l^{\infty}\left(\mathbf{Z}^{2}\right) \text { and } \lambda \in \mathbf{R},
$$

that is, $G$ commutes with the addition of constants. Now, it is a simple fact that order-preserving mappings commuting with the addition of constants are nonexpansive in $l^{\infty}\left(\mathbf{Z}^{2}\right)$ (M. G. Crandall and L. Tartar [5]). Indeed, if $U, V \in C$, then $U \leqslant V+\lambda$ with $\lambda=\left\|(U-V)^{+}\right\|_{\infty}$. But $V+\lambda \in C$, and thus (using monotonicity) we deduce

$$
\vec{G}(U) \leqslant \vec{G}(V+\lambda)=\vec{G}(V)+\lambda, \text { so }\left\|(\vec{G}(U)-\vec{G}(V))^{+}\right\|_{\infty} \leqslant\left\|(U-V)^{+}\right\|_{\infty},
$$

which implies, in particular, the nonexpansiveness on $C$.

Another simple property of $\vec{G}$ is that it commutes with translations, i.e. if $l, m \in \mathbf{Z}$ are fixed and $\tau_{l, m}$ is the linear mapping defined by $\left(\tau_{l, m} U\right)_{j, k}=U_{j+l, k+m}$, then we have $\tau_{l, m} \vec{G}(U)=\vec{G}\left(\tau_{l, m} U\right)$ for all $V \in l^{\infty}\left(\mathbf{Z}^{2}\right)$. Using this property in conjunction with (3.2), we can deduce

$$
\left\|\Delta_{+}^{x} \vec{G}(U)\right\|_{\infty} \leqslant\left\|\Delta_{+}^{x} U\right\|_{\infty}, \quad\left\|\Delta_{+}^{y} \vec{G}(U)\right\|_{\infty} \leqslant\left\|\Delta_{+}^{y} U\right\|_{\infty}
$$

for all $U \in C$. For example,

$$
\begin{aligned}
\left\|\Delta_{+}^{x} \vec{G}(U)\right\|_{\infty} & =\left\|\tau_{1,0} \vec{G}(U)-\vec{G}(U)\right\|_{\infty}=\left\|\vec{G}\left(\tau_{1,0} U\right)-\vec{G}(U)\right\| \\
& \leqslant\left\|\tau_{1,0} U-U\right\|_{\infty}=\left\|\Delta_{+}^{x} U\right\|_{\infty},
\end{aligned}
$$

since $\tau_{l, m}$ leaves invariant $C$ for any $l, m \in \mathbf{Z}$. An immediate consequence of (3.3) is that $\vec{G}$ leaves $C$ invariant $(\vec{G}(C) \subset C)$.

The last property we observe is the following: If $n, j \geqslant 0$ and $U \in C$, then

$$
\begin{aligned}
&\left\|\vec{G}^{n+j}(U)-\vec{G}^{n}(U)\right\|_{\infty} \leqslant\left\|\vec{G}^{j}(U)-U\right\|_{\infty} \\
& \leqslant \sum_{l=0}^{j-1}\left\|\vec{G}^{j-l}(U)-\vec{G}^{j-(l+1)}(U)\right\|_{\infty} \leqslant j\|\vec{G}(U)-U\|_{\infty},
\end{aligned}
$$

and from the explicit form (3) we finally obtain

$$
\left\|\vec{G}^{n+j}(U)-\vec{G}^{n}(U)\right\|_{\infty} \leqslant j \Delta t K \text { for } n, j \geqslant 0, U \in C,
$$

where $K$ is given by

$$
K=\sup \left\{|g(\xi)| ; 0 \leqslant l \leqslant p+q+1,0 \leqslant m \leqslant r+s+1,\left|\xi_{l, m}\right| \leqslant R\right\},
$$

and $\xi$ denotes a vector with components $\xi_{l, m}$.

We record all these properties in

Proposition 3.1. Let $R>0$ and $C$ be defined as above. Let the scheme be monotone on $[-R, R]$ and $\vec{G}: C \rightarrow l^{\infty}\left(\mathbf{Z}^{2}\right)$ be given by (3) with $g$ bounded on bounded sets. Then we have:

(i) $\vec{G}(U) \leqslant \vec{G}(V)$ for $U, V \in C, U \leqslant V$;

(ii) $\vec{G}(U+\lambda)=\vec{G}(U)+\lambda$ for $U \in C, \lambda \in \mathbf{R}$; 
(iii) $\|\vec{G}(U)-\vec{G}(V)\|_{\infty} \leqslant\|U-V\|_{\infty}$ for $U, V \in C$;

(iv) $\left\|\Delta_{+}^{x} \vec{G}(U)\right\|_{\infty} \leqslant\left\|\Delta_{+}^{x} U\right\|_{\infty},\left\|\Delta_{+}^{y} \vec{G}(U)\right\|_{\infty} \leqslant\left\|\Delta_{+}^{y} U\right\|_{\infty}$ for $U \in C$;

(v) $\vec{G}(C) \subset C$;

(vi) $\left\|\vec{G}^{n+j}(U)-\vec{G}^{n}(U)\right\|_{\infty} \leqslant j \Delta t K$ for $U \in C, n, j \geqslant 0$,

where $K$ is given by (3.5);

(vii) $\left\|\vec{G}^{n}(U)\right\|_{\infty} \leqslant\|U\|_{\infty}+n \Delta t \Lambda$, for $U \in C$,

where $\Lambda=|g(0, \ldots, 0)|=|H(0,0)|$.

The only new property is (vii), which is easily proved by first observing that $G^{n}(0)=-n \Delta t g(0,0, \ldots, 0)$ and so

$$
\left\|G^{n}(U)\right\|_{\infty} \leqslant\left\|G^{n}(U)-G^{n}(0)\right\|_{\infty}+\left\|G^{n}(0)\right\|_{\infty} \leqslant\|U\|_{\infty}+n \Delta t \Lambda .
$$

4. Proof of Theorem 1. The proof of Theorem 1 given here is related to the proof of uniqueness of viscosity solutions of (IVP) presented in [1] and it also involves estimates introduced in [2]. Throughout this section we will assume the hypotheses and notation of Theorem 1 . In addition, we will at first assume that

$$
\begin{cases}u(x, y, t) \rightarrow 0 & \text { as }|x|+|y| \rightarrow \infty, \\ U_{j, k}^{n} \rightarrow 0 & \text { as }|j|+|k| \rightarrow \infty\end{cases}
$$

hold uniformly for bounded $t, n \Delta t \geqslant 0$. This assumption allows a simplified presentation and is easily relaxed later. Moreover, (4.1) holds if $u_{0} \rightarrow 0$ as $|x|+|y|$ $\rightarrow \infty$.

It will be convenient to define

$$
Q=\mathbf{R}^{2} \times[0, \infty), \quad Q_{T}=\mathbf{R}^{2} \times[0, T],
$$

and the discrete analogues

$$
\begin{aligned}
& Q^{d}=\Delta \times\left(\Delta t \mathbf{Z}^{+}\right)=\left\{\left(x_{j}, y_{k}, n \Delta t\right): j, k=0, \pm 1, \ldots ; n=0,1, \ldots,\right\}, \\
& Q_{N}^{d}=\left\{\left(x_{j}, y_{k}, n \Delta t\right) \in Q^{d}: n \leqslant N\right\} .
\end{aligned}
$$

Hereafter $T>0$ and $N \in \mathbf{Z}^{+}$satisfying

$$
(N-1) \Delta t<T \leqslant N \Delta t
$$

are fixed. We seek to estimate $u\left(x_{j}, y_{k}, n \Delta t\right)-U_{j, k}^{n}$. To this end, we will assume

$$
\sup _{\substack{j, k \in \mathbf{Z} \\ 0 \leqslant n \leqslant N}}\left(u\left(x_{j}, y_{k}, n \Delta t\right)-U_{j, k}^{n}\right)=\sigma>0
$$

and then produce an upper bound on $\sigma$. In exactly the same way, if $\inf \left(u\left(x_{j}, y_{k}, n \Delta t\right)-U_{j, k}^{n}\right)=-\sigma<0$, we could estimate $\sigma$ and the conjunction of these estimates bounds $\left(u\left(x_{j}, y_{k}, n \Delta t\right)-U_{j, k}^{n}\right)$.

We are going to define a function $\psi: Q \times Q^{d} \rightarrow \mathbf{R}$ which is a principal ingredient in the proof. This function depends on $\sigma$ and $T$ above as well as

$$
M=\sup _{|\xi| \in \mathbf{R}^{2}}\left(\left|u_{0}(\xi)\right|+T|H(0,0)|\right)+1
$$


a positive parameter $\varepsilon$, and a function $\beta: \mathbf{R}^{2} \times \mathbf{R} \rightarrow \mathbf{R}$ as follows: For $(\xi, t) \in Q$ and $(\eta, s) \in Q^{d}$,

$$
\begin{array}{r}
\psi(\xi, t, \eta, s)=u(\xi, t)-U_{j, k}^{n}-\frac{\sigma}{4 T}(t+s)+\left(5 M+\frac{\sigma}{2}\right) \beta_{\varepsilon}(\xi-\eta, t-s), \\
\text { where }(\eta, s)=\left(x_{j}, y_{k}, n \Delta t\right) \text { and } \beta_{\varepsilon}(\xi, t)=\beta(\xi / \varepsilon, t / \varepsilon) .
\end{array}
$$

We remark that (4.6) guarantees

$$
|u| \leqslant M \text { on } Q_{T} \quad \text { and } \quad\left\|U^{n}\right\|_{\infty} \leqslant M \text { for } 0 \leqslant n \leqslant N
$$

see Proposition 1.1(iii) and Proposition 3.1(vii). The function $\beta$ of (4.7) will satisfy

$$
\left\{\begin{array}{l}
\beta \text { is smooth on } \mathbf{R}^{2} \times \mathbf{R}, \quad 0 \leqslant \beta \leqslant 1, \beta(0,0)=1, \\
\beta(\xi, t)=0 \text { if }|\xi|^{2}+t^{2}>1,
\end{array}\right.
$$

as well as other conditions imposed later. The next step is to maximize $\psi$ over $Q_{T, N}=Q_{T} \times Q_{N}^{d}$.

LEMMA 4.1. Under the above assumptions there is a point $\left(\xi_{0}, t_{0}, \eta_{0}, s_{0}\right) \in Q_{T, N}$ such that

(i) $\psi\left(\xi_{0}, t_{0}, \eta_{0}, s_{0}\right) \geqslant \psi(\xi, t, \eta, s)$ for $(\xi, t, \eta, s) \in Q_{T, N}$ and

(ii) $\beta_{\varepsilon}\left(\xi_{0}-\eta_{0}, t_{0}-s_{0}\right) \geqslant 3 / 5$.

Proof. The existence of $\left(\xi_{0}, t_{0}, \eta_{0}, s_{0}\right)$ follows from the fact that if $\left(\xi^{l}, t^{l}, \eta^{l}, s^{l}\right) \in$ $Q_{T, N}$ and

$$
\psi\left(\xi^{l}, t^{l}, \eta^{l}, s^{l}\right) \rightarrow \sup _{Q_{T, N}} \psi
$$

then $\left(\xi^{l}, t^{l}, \eta^{l}, s^{l}\right)$ remains bounded. Indeed, from (4.5), (4.7), (4.9), one sees that

$$
\sup _{Q_{T, N}} \psi \geqslant \sup _{\substack{j, k \in \mathbf{Z} \\ 0 \leqslant n \leqslant N}}\left(u\left(x_{j}, y_{k}, n \Delta t\right)-U_{j, k}^{n}\right)+5 M=\sigma+5 M,
$$

while (4.7), (4.8) imply

$$
\Psi(\xi, t, \eta, s) \leqslant 2 M \text { if } \beta_{\varepsilon}(\xi-\eta, t-s)=0 .
$$

Hence (4.10) implies $\beta_{\varepsilon}\left(\xi^{l}-\eta^{l}, t^{l}-s^{l}\right)>0$ for large $l$, and so $\left(\xi^{l}-\eta^{l}\right)^{2}+$ $\left(t^{\prime}-s^{l}\right)^{2}<\varepsilon^{2}$. From this, (4.1) and (4.7) we see that if $\left|\xi^{\prime}\right|+\left|\eta^{\prime}\right| \rightarrow \infty$, then

$$
\underset{l \rightarrow \infty}{\limsup } \Psi\left(\xi^{l}, t^{l}, \eta^{l}, s^{l}\right) \leqslant 5 M+\sigma / 2 .
$$

This contradicts (4.10), (4.11).

To prove (ii), observe that

$$
2 M+(5 M+\sigma / 2) \beta_{\varepsilon}\left(\xi_{0}-\eta_{0}, t_{0}-s_{0}\right) \geqslant \psi\left(\xi_{0}, t_{0}, \eta_{0}, s_{0}\right) \geqslant \sup \Psi \geqslant 5 M+\sigma,
$$

so

$$
\beta_{\varepsilon}\left(\xi_{0}-\eta_{0}, t_{0}-s_{0}\right) \geqslant(6 M+2 \sigma) /(10 M+\sigma) \geqslant 3 / 5 .
$$

In what follows we will put

$$
\varepsilon=(\Delta x+\Delta y+\Delta t)^{1 / 4}=\left(\lambda^{x}+\lambda^{y}+1\right)^{1 / 4}(\Delta t)^{1 / 4},
$$

although we will not use this relation except at certain points in the argument. 
There are now several cases to be considered. These are: $t_{0}, s_{0}>0 ; t_{0} \geqslant 0, s_{0}=0$; and $t_{0}=0, s_{0}>0$. We begin with the case $t_{0}, s_{0}>0$.

1st case. $t_{0}>0, s_{0}>0$. It follows from Lemma 4.1(i) that $\left(\xi_{0}, t_{0}\right)$ is a maximum point on $Q_{T}$ of

$$
(\xi, t) \rightarrow u(\xi, t)-\sigma t / 4+(5 M+\sigma / 2) \beta_{\varepsilon}\left(\xi-\eta_{0}, t-s_{0}\right) .
$$

By the definition of viscosity solutions and the equation solved by $u$, we therefore have

$$
\begin{aligned}
\frac{\sigma}{4}-\left(5 M+\frac{\sigma}{2}\right) & D_{t} \beta_{\varepsilon}\left(\xi-\eta_{0}, t_{0}-s_{0}\right) \\
& +H\left(-\left(5 M+\frac{\sigma}{2}\right) D_{\xi} \beta_{\varepsilon}\left(\xi_{0}-\eta_{0}, t_{0}-s_{0}\right)\right) \leqslant 0 .
\end{aligned}
$$

(Here and below, $D_{t} \beta_{\varepsilon}, D_{\xi} \beta_{\varepsilon}$ stand for the indicated derivatives of $\beta_{\varepsilon}(\xi, t)$ which are then evaluated at the point shown.)

The analogous estimate on the discrete side requires more work. Let

$$
\left(\eta_{0}, s_{0}\right)=\left(\left(x_{j_{0}}, y_{k_{0}}\right), n_{0} \Delta t\right)
$$

then $\left(j_{0}, k_{0}, n_{0}\right)$ minimizes

$$
(j, k, n) \rightarrow U_{j, k}^{n}+\frac{\sigma}{4}(n \Delta t)-\left(5 M+\frac{\sigma}{2}\right) \beta_{\varepsilon}\left(\xi_{0}-\left(x_{j}, y_{k}\right), t_{0}-n \Delta t\right)
$$

over $\left(x_{j}, y_{k}, n \Delta t\right) \in Q_{N}^{d}$. Thus

$$
\begin{aligned}
U_{j, k}^{n} \geqslant & U_{j_{0}, k_{0}}^{n_{0}}+(\sigma / 4)\left(n_{0}-n\right) \Delta t-(5 M+\sigma / 2) \\
& \times\left(\beta_{\varepsilon}\left(\xi_{0}-\eta_{0}, t_{0}-s_{0}\right)-\beta_{\varepsilon}\left(\xi_{0}-(j \Delta x, k \Delta y), t_{0}-n \Delta t\right)\right)
\end{aligned}
$$

for $0 \leqslant n \leqslant N$ and all $j, k$. We next use the monotonicity of $G$ and (4.15) with $n=n_{0}-1$ to conclude that

$$
\begin{aligned}
& U_{j_{0}, k_{0}}^{n_{0}}=\vec{G}\left(U^{n_{0}-1}\right)_{j_{0}, k_{0}} \\
& \geqslant U_{j_{0}, k_{0}}^{n_{n_{0}}}+\frac{\sigma}{4} \Delta t-\left(5 M+\frac{\sigma}{2}\right)\left(\beta_{\varepsilon}\left(\xi_{0}-\eta_{0}, t_{0}-s_{0}\right)-\beta_{\varepsilon}\left(\xi_{0}-\eta_{0}, t_{0}-s_{0}+\Delta t\right)\right) \\
& -\Delta \operatorname{tg}\left(\frac{5 M+\sigma / 2}{\Delta x} \Delta_{+}^{x} \beta_{\varepsilon}\left(\xi_{0}-\left(\left(j_{0}-p\right) \Delta x,\left(k_{0}-r\right) \Delta y\right), t_{0}-s_{0}+\Delta t\right),\right. \\
& \left.\quad \ldots, \frac{5 M+\sigma / 2}{\Delta y} \Delta_{+}^{y} \beta_{\varepsilon}\left(\xi_{0}-\left(\left(j_{0}-p\right) \Delta x,\left(k_{0}-r\right) \Delta y\right), t_{0}-s_{0}+\Delta t\right), \ldots\right) .
\end{aligned}
$$

To guarantee the validity of this step we must show that the arguments of $g$ above lie in $[-(L+1), L+1]$, since the monotonicity is only assumed in this case. Consider a typical argument of $g$ above; e.g., any of the $x$-differences has the form

$$
\begin{aligned}
\frac{5 M+\sigma / 2}{\Delta x}\left[\beta _ { \varepsilon } \left(\xi_{0}-\eta_{0}-\right.\right. & \left.((l+1) \Delta x, m \Delta y), t_{0}-s_{0}+\Delta t\right) \\
& \left.-\beta_{\varepsilon}\left(\xi_{0}-\eta_{0}-(l \Delta x, m \Delta y), t_{0}-s_{0}+\Delta t\right)\right]
\end{aligned}
$$

where $l, m$ are bounded integers,

$$
1+|l|, \quad|m| \leqslant \max (p, r, q+1, s+1)+1=K .
$$


Clearly, the difference between (4.17) and $-(5 M+\sigma / 2) D_{1} \beta_{\varepsilon}\left(\xi_{0}-\eta_{0}, t_{0}-s_{0}\right)$, where $D_{1}$ denotes differentiation in the first spatial argument, is estimated in the form const $\varepsilon^{-2}(\Delta x+\Delta y+\Delta t)$, where the constant involves bounds on the second derivative of $\beta$ but is independent of $\varepsilon$. Invoking (4.12), we find these errors to be at most

$$
\operatorname{const}(\Delta t)^{1 / 2} \text {, }
$$

so (4.17) differs from $-(5 M+\sigma / 2) D_{1} \beta_{\varepsilon}\left(\zeta_{0}-\eta_{0}, t_{0}-s_{0}\right)$ by at most 1 if $\Delta t$ is sufficiently small. Using the next lemma and these remarks, we see that the arguments of $g$ in (4.16) lie in $[-(L+1), L+1]$ if $\Delta t$ is sufficiently small. (Parts (ii) and (iii) of the lemma are used later.)

LEMMA 4.2. Let $\left(\xi_{0}, t_{0}, \eta_{0}, s_{0}\right)$ be as in Lemma 4.1 and $L$ be the Lipschitz constant of $u_{0}$. Then

(i) $(5 M+\sigma / 2)\left|D_{\xi} \beta_{\varepsilon}\left(\xi_{0}-\eta_{0}, t_{0}-s_{0}\right)\right| \leqslant L$.

Let $L_{1}=\max (|H(p)|:|p| \leqslant L)$ and $t_{0}>0$. Then

(ii) $-(5 M+\sigma / 2) D_{t} \beta_{\varepsilon}\left(\xi_{0}-\eta_{0}, t_{0}-s_{0}\right) \leqslant L_{1}-\sigma / 4 T$.

If also $T>t_{0}$, then

(iii) $(5 M+\sigma / 2)\left|D_{t} \beta_{\varepsilon}\left(\xi_{0}-\eta_{0}, t_{0}-s_{0}\right)\right| \leqslant L_{1}+\sigma / 4 T$.

Proof. By assumption, the mapping

$$
\xi \rightarrow u\left(\xi, t_{0}\right)+(5 M+\sigma / 2) \beta_{\varepsilon}\left(\xi-\eta_{0}, t_{0}-s_{0}\right)
$$

is maximized at $\xi=\xi_{0}$. Thus for $\xi \in \mathbf{R}^{2}$

$$
\begin{gathered}
(5 M+\sigma / 2)\left(\beta\left(\xi-\eta_{0}, t_{0}-s_{0}\right)-\beta\left(\xi_{0}-\eta_{0}, t_{0}-s_{0}\right)\right) \\
\leqslant u\left(\xi_{0}, t_{0}\right)-u\left(\xi, t_{0}\right) \leqslant L\left|\xi_{0}-\xi\right|,
\end{gathered}
$$

where the last inequality is from Proposition 1.1(v). The inequality (i) follows at once. Similarly,

$$
t \rightarrow u\left(\xi_{0}, t\right)-\sigma t / 4+(5 M+\sigma / 2) \beta_{\varepsilon}\left(\xi_{0}-\eta_{0}, t-s_{0}\right)
$$

is maximized over $[0, T]$ at $t_{0}>0$, so for small $h>0$

$$
\begin{array}{r}
(5 M+\sigma / 2)\left[\beta_{\varepsilon}\left(\xi_{0}-\eta_{0}, t_{0}-h-s_{0}\right)-\beta_{\varepsilon}\left(s_{0}-\eta_{0}, t_{0}-s_{0}\right)\right] \\
\leqslant\left(u\left(\xi_{0}, t_{0}\right)-u\left(\xi_{0}, t_{0}-h\right)\right)-\frac{\sigma h}{4 T} \leqslant L_{1} h-\frac{\sigma}{4 T} h,
\end{array}
$$

where the last inequality is from Proposition 1.1(v). The inequality (ii) follows at once. If also $T>t_{0}$, then one makes the two-sided estimate in the obvious way.

Now we return to (4.16). In this expression we replace each difference in the arguments of $g$ by the corresponding derivative of $-(5 M+\sigma / 2) \beta_{\varepsilon}\left(\xi-\eta_{0}, t_{0}-s_{0}\right)$ at $\xi=\xi_{0}$, thereby creating errors we can estimate-using the locally Lipschitz property of $g$-by multiples of $(\Delta x+\Delta y+\Delta t) \varepsilon^{-2}$. Then we use the consistency of the numerical Hamiltonian $g$ with $H$ to conclude that

$$
\begin{aligned}
\frac{\sigma}{4} \leqslant & \left(5 M+\frac{\sigma}{2}\right) \frac{\beta_{\varepsilon}\left(\xi_{0}-\eta_{0}, t_{0}-s_{0}\right)-\beta_{\varepsilon}\left(\xi_{0}-\eta_{0}, t_{0}-\left(s_{0}-\Delta t\right)\right)}{\Delta t} \\
& +H\left(-\left(5 M+\frac{\sigma}{2}\right) D_{\xi} \beta_{\varepsilon}\left(\xi_{0}-\eta_{0}, t_{0}-s_{0}\right)\right)+C\left(\frac{\Delta x+\Delta y+\Delta t}{\varepsilon^{2}}\right),
\end{aligned}
$$


where $C$ is a constant one could easily estimate. Making similar arguments on the $t$-difference above, we further deduce that

$$
\begin{aligned}
\frac{\sigma}{4} \leqslant & -\left(5 M+\frac{\sigma}{2}\right) D_{t} \beta_{\varepsilon}\left(\xi_{0}-\eta_{0}, t_{0}-s_{0}\right) \\
& +H\left(-\left(5 M+\frac{\sigma}{2}\right) D_{\xi} \beta_{\varepsilon}\left(\xi_{0}-\eta_{0}, t_{0}-s_{0}\right)\right)+C\left(\frac{\Delta x+\Delta y+\Delta t}{\varepsilon^{2}}\right) .
\end{aligned}
$$

Taken together, (4.13) and (4.20) yield

$$
\sigma \leqslant C\left(\frac{\Delta x+\Delta y+\Delta t}{\varepsilon^{2}}\right)
$$

(with a new constant $C$ ). We again invoke (4.12) so that this becomes

$$
\sigma \leqslant C(\Delta x+\Delta y+\Delta t)^{1 / 2}=C\left(\lambda^{x}+\lambda^{y}+1\right)^{1 / 2}(\Delta t)^{1 / 2},
$$

which establishes the desired estimate.

We turn to the cases in which one of $t_{0}$ or $s_{0}$ is 0 . In these cases we do not need to use the information that $u$ is a solution or the detailed properties of $G$. We rely on (4.11) and simple considerations of continuity. However, we will restrict $\beta$ to satisfy

$$
\begin{cases}\beta(\xi, t)=1-\left(|\xi|^{2}+t^{2}\right) & \text { for }|\xi|^{2}+t^{2}<\frac{1}{2} \\ \beta(\xi, t)<\frac{1}{2} & \text { for }|\xi|^{2}+t^{2}>\frac{1}{2}\end{cases}
$$

In the event (4.21) holds we know from Lemma 4.1(ii) that $\left|\xi_{0}-\eta_{0}\right|^{2}+\left(t_{0}-s_{0}\right)^{2}<$ $\frac{1}{2} \varepsilon^{2}$. Hence

$$
\begin{aligned}
& D_{\xi} \beta_{\varepsilon}\left(\xi_{0}-\eta_{0}, t_{0}-s_{0}\right)=-\frac{2}{\varepsilon^{2}}\left(\xi_{0}-\eta_{0}\right), \\
& D_{t} \beta_{\varepsilon}\left(\xi_{0}-\eta_{0}, t_{0}-s_{0}\right)=-\frac{2}{\varepsilon^{2}}\left(t_{0}-s_{0}\right),
\end{aligned}
$$

and then from Lemma 4.2 we conclude that:

$$
\begin{gathered}
\left|\xi_{0}-\eta_{0}\right| \leqslant \varepsilon^{2} L /(10 M+\sigma) \\
\text { If } t_{0}=T, \text { then }\left|t_{0}-s_{0}\right| \leqslant \frac{\varepsilon^{2}\left(L_{1}-\sigma / 4 T\right)}{10 M+\sigma} ; \\
\text { If } T>t_{0}>0, \text { then }\left|t_{0}-s_{0}\right| \leqslant \frac{\varepsilon^{2}\left(L_{1}+\sigma / 4 T\right)}{10 M+\sigma} .
\end{gathered}
$$

2nd case. $t_{0} \geqslant 0, s_{0}=0$.

By (4.11), Proposition 1.1(v) and the choice of $U^{0}$,

$$
\begin{aligned}
5 M+\sigma \leqslant & \psi\left(\xi_{0}, t_{0}, \eta_{0}, 0\right) \leqslant\left|u\left(\xi_{0}, t_{0}\right)-u\left(\eta_{0}, t_{0}\right)\right|+\left|u\left(\eta_{0}, t_{0}\right)-u\left(\eta_{0}, 0\right)\right| \\
& +(5 M+\sigma / 2) \beta_{\varepsilon}\left(\xi_{0}-\eta_{0}, t_{0}-s_{0}\right) \\
\leqslant & L\left|\xi_{0}-\eta_{0}\right|+L_{1} t_{0}+5 M+\sigma / 2 .
\end{aligned}
$$

The estimate (4.22) holds and either $t_{0}=0$ or one of (4.23), (4.24) holds. The above thus yields $\sigma \leqslant$ const $\varepsilon^{2}$, and again we are done. 
3rd case. $t_{0}=0, s_{0}>0$. In a manner similar to the above we find (using also Proposition 3.1(v)) that

$$
5 M+\sigma \leqslant \psi\left(\xi_{0}, 0, \eta_{0}, s_{0}\right) \leqslant L\left|\xi_{0}-\eta_{0}\right|+K s_{0}+5 M+\sigma / 2
$$

and so

$$
\sigma \leqslant 2 L\left|\xi_{0}-\eta_{0}\right|+2 K s_{0} .
$$

We now need to estimate $s_{0}$ suitably and invoke (4.22) once more to complete the proof.

From

$$
\psi\left(\xi_{0}, t_{0}, \eta_{0}, s_{0}\right)=\psi\left(\xi_{0}, 0, \eta_{0}, s_{0}\right) \geqslant \psi\left(\xi_{0}, 0, \eta_{0}, s_{0}-\Delta t\right),
$$

we conclude that

$$
\begin{aligned}
-U_{j_{0}, k_{0}}^{n_{0}}- & \frac{\sigma}{4 T} s_{0}+\left(5 M+\frac{\sigma}{2}\right) \beta_{\varepsilon}\left(\xi_{0}-\eta_{0},-s_{0}\right) \\
& \geqslant-U_{j_{0}, k_{0}}^{n_{0}-1}-\frac{\sigma}{4 T}\left(s_{0}-\Delta t\right)+\left(5 M+\frac{\sigma}{2}\right) \beta_{\varepsilon}\left(\xi_{0}-\eta_{0},-s_{0}+\Delta t\right),
\end{aligned}
$$

where the notation (4.14) is being used. Since

$$
\beta_{\varepsilon}\left(\xi_{0}-\eta_{0}, t_{0}-s_{0}\right)=\beta_{\varepsilon}\left(\xi_{0}-\eta_{0},-s_{0}\right) \geqslant 3 / 5
$$

by Lemma 4.1 and (4.12), (4.21) hold, we can assume that $\beta_{\varepsilon}$ has the quadratic form (4.12) by taking $\Delta t$ small. The above then becomes

$$
\frac{5 M+\sigma / 2}{\varepsilon^{2}}\left(s_{0}^{2}-\left(s_{0}-\Delta t\right)^{2}\right) \leqslant U_{j_{0}, k_{0}}^{n_{0}-1}-U_{j_{0}, k_{0}}^{n_{0}}-\frac{\sigma}{4 T} \Delta t \leqslant K \Delta t .
$$

Analyzing this inequality, we conclude that $s_{0} \leqslant \operatorname{const}\left(\varepsilon^{2}+\Delta t\right)$. Using this in the estimate on $\sigma$ above yields $\sigma \leqslant \operatorname{const}\left(\varepsilon^{2}+\Delta t\right)$, and again $\varepsilon=(\Delta x+\Delta y+\Delta t)^{1 / 4}$ yields $\sigma \leqslant \operatorname{const}(\Delta x+\Delta y+\Delta t)^{1 / 2}$.

Proof of the General Case. The remaining step is to remove the restriction (4.1). There are two possible ways to do this. We may, for example, follow the uniqueness proof in [1] and replace $\psi$ by

$$
\Phi=\psi+2 \delta \zeta(\xi, t, \eta, s),
$$

where $\delta>0, \zeta=\tilde{\zeta}\left(\xi+\xi_{0}, t+t_{0}, y+y_{0}, s+s_{0}\right)$ and $\tilde{\zeta} \in C_{0}^{\infty}\left(\mathbf{R}^{2} \times \mathbf{R} \times \mathbf{R}^{2} \times \mathbf{R}\right)$, $0 \leqslant \tilde{\zeta} \leqslant 1, \tilde{\zeta}(0)=1$ and $\left(\xi_{0}, t_{0}, \eta_{0}, s_{0}\right)$ is a point such that

$$
\psi\left(\xi_{0}, t_{0}, \eta_{0}, s_{0}\right) \geqslant \sup \psi-\delta .
$$

Then adapting the above proof, one reaches the desired conclusion. (See also [11].) Another argument makes use of the hyperbolic nature of the problem, namely the finite speed of propagation. Observe that, without loss of generality, we may assume $H(0)=0$ (replace $u(x, t)$ by $u(x, t)+t H(0), H$ by $H-H(0), g$ by $g-H(0), U^{n}$ by $\left.U^{n}+n \Delta t\right)$. In the statement of the next result, which was proved in [2], we use the notation $B(x, R)$ for the closed ball in $\mathbf{R}^{N}$ with center $x$ and radius $R$ and put $B_{R}=B(0, R)$.

TheOREM. Let $u_{0}, v_{0} \in W^{1, \infty}\left(\mathbf{R}^{N}\right)$, let $H \in W_{\text {loc }}^{1, \infty}\left(\mathbf{R}^{N}\right)$, and denote the semigroup solving (IVP) by $S(t)$. Then, if $y_{0} \in \mathbf{R}^{N}$ and $u_{0}(y)=v_{0}(y)$ on $B\left(y_{0}, R\right)$, we have

$$
\left(S(t) u_{0}\right)(x)=\left(S(t) v_{0}\right)(x) \text { on } B\left(y_{0}, R-v t\right) \text {, }
$$

where $\nu=\left\|H^{\prime}\right\|_{L^{\infty}\left(B_{r}\right)}$ and $r=\max \left(\left\|D u_{0}\right\|_{L^{\infty}},\left\|D v_{0}\right\|_{L^{\infty}}\right)$. 
On the other hand, it is clear from the definitions that if $U_{j, k}=V_{j, k}$ for $\left|j-j_{0}\right| \leqslant R$, $\left|k-k_{0}\right| \leqslant R(R \in \mathbf{N})$, then $U_{j, k}^{n}=V_{j, k}^{n}$ for $\left|j-j_{0}\right| \leqslant R-K n,\left|k-k_{0}\right| \leqslant R-K n$ with $K=\max (p, r, q+1, s+1)$. Thus if $u_{0} \equiv v_{0}$ in $B\left(\xi_{0}, R\right)$ and $\xi_{0}=\left(x_{0}, y_{0}\right)$, and if $U^{n}, V^{n}$ are the discrete approximations generated by our scheme, we see that $U_{j, k}^{n}=V_{j, k}^{n}$ for $j, k$ satisfying

$$
\left|j-x_{0} / \Delta x\right| \leqslant R / \Delta x-2-K n, \quad\left|k-y_{0} / \Delta y\right| \leqslant R / \Delta y-2-K n,
$$

i.e.,

$$
\begin{aligned}
& \left|j \Delta x-x_{0}\right| \leqslant(R-2 \Delta x)-\frac{K}{\lambda^{x}}(n \Delta t), \\
& \left|k \Delta y-y_{0}\right| \leqslant(R-2 \Delta y)-\frac{K}{\lambda^{y}}(n \Delta t) .
\end{aligned}
$$

It is then easy to conclude the argument by remarking that, uniformly in $z_{0} \in \mathbf{R}^{2}$, we may find $R$ large enough and a Lipschitz continuous $v_{0}$ with compact support such that: $u_{0}=v_{0}$ on $B\left(z_{0}, R\right) ;\left(S(t) u_{0}\right)(z)=\left(S(t) v_{0}\right)(z)$ for $\left|z-z_{0}\right| \leqslant 1,0 \leqslant t \leqslant$ $T$; and $U_{j, k}^{n}=V_{j, k}^{n}$ for $\left|(j \Delta x, k \Delta y)-z_{0}\right| \leqslant 1,0 \leqslant n \leqslant N$. Applying the result already proved (as we may, since $V^{n}, S(t) v_{0}$ also have compact support), Theorem 1 is proved.

We pause to comment on a few of the possible extensions of the preceding results. First of all, it is straightforward to treat more general Hamiltonians $H(x, t, u, D u)$. For example, in one space dimension, let $H(x, t, r)$ be Lipschitz continuous in $\mathbf{R} \times[0, T] \times[-R, R]$ for each $T, R>0$. Then an approximation

$$
\begin{aligned}
U_{j}^{n+1} & =U_{j}^{n}-\Delta \operatorname{tg}\left(x_{j}, t_{n}, \frac{\Delta_{+} U_{j-p}^{n}}{\Delta x}, \ldots, \frac{\Delta_{+} U_{j+q}^{n}}{\Delta x}\right) \\
& =G\left(x_{j}, t_{n}, U_{j-p}^{n}, \ldots, U_{j+q+1}^{n}\right)
\end{aligned}
$$

is consistent if $g(x, t, \alpha, \ldots, \alpha)=H(x, t, \alpha)$ and monotone if $U_{j}^{n+1}$ is a nondecreasing function of $U_{j-p}^{n}, \ldots, U_{j+q+1}^{n}$. If the numerical Hamiltonian is also Lipschitz continuous on bounded sets of $\mathbf{R} \times[0, T] \times \mathbf{R}^{p+q+2}$, we can again estimate $U_{j}^{n}-$ $u\left(x_{j}, t_{n}\right)$ by a multiple of $(\Delta t)^{1 / 2}$ if $u_{0}$ is Lipschitz continuous. (The simple Propositions 1.1 and 3.1 need to be appropriately generalized. See [11].)

Next, we could discuss the corresponding stationary problem $u+H(D u)=f(x)$ in $\mathbf{R}^{N}$ as well as boundary value problems (see [9]), but we will not formulate any precise results here. It is also clear that implicit approximations can be handled equally well.

We conclude this section with some final remarks in the context of the equation $u_{t}+H(D u)=0$ (which apply to its generalizations as well). If one reexamines the above proofs under the assumption that $H$ and $g$ are globally Lipschitz continuous, one sees that the estimate on $U_{j, k}^{n}-u\left(x_{j}, y_{k}, n \Delta t\right)$ depends on $u_{0}$ through its Lipschitz constant $L=L\left(u_{0}\right)$ (provided that $u_{0}$ is kept bounded) in the form

$$
\left|U_{j, k}^{n}-u\left(x_{j}, y_{k}, n \Delta t\right)\right| \leqslant C\left(L^{2}(\Delta t)^{1 / 2}\right)
$$

(where we assume $L$ is not small and $\Delta t$ is not large). Using this fact and the nonexpansive nature of $S(t)$ (Proposition 1.1(ii)) and $\vec{G}$ (Proposition 3.1(iii)), we 
conclude that if $U_{j, k}^{n}, u, V_{j, k}^{n}, v$, are the discrete and exact solutions for initial data $u_{0}$ (possibly not Lipschitz) and $v_{0}$ (Lipschitz), then

$$
\begin{aligned}
\left|U_{j, k}^{n}-u\left(x_{j}, y_{k}, t_{n}\right)\right| \leqslant & \left|U_{j, k}^{n}-V_{j, k}^{n}\right|+\left|V_{j, k}^{n}-v\left(x_{j}, y_{k}, t_{n}\right)\right| \\
& +\left|v\left(x_{j}, y_{k}, t_{n}\right)-u\left(x_{j}, y_{k}, t_{n}\right)\right| \\
\leqslant & 2\left\|u_{0}-v_{0}\right\|+C\left(L\left(v_{0}\right)\right)^{2}(\Delta t)^{1 / 2} .
\end{aligned}
$$

This allows us to conclude the convergence of the numerical scheme for general $u_{0} \in \mathrm{BUC}\left(\mathbf{R}^{N}\right)$ with an error estimate. For example, if $u_{0}$ is Hölder continuous with exponent $\alpha$, we can choose $v_{0}$ above so that the error is at most $\operatorname{const}(\Delta t)^{\alpha /(2(2-\alpha))}$.

5. Convergence of the Vanishing Viscosity Method. It has long been standard to attempt to approximate (IVP) by the problem

$$
\begin{array}{ll}
\partial u^{\varepsilon} / \partial t+H\left(D u^{\varepsilon}\right)-\varepsilon \Delta u^{\varepsilon}=0 & \text { in } \mathbf{R}^{N} \times(0, \infty), \\
u^{\varepsilon}(x, 0)=u_{0}(x) & \text { in } \mathbf{R}^{N} .
\end{array}
$$

By anology with fluid mechanics, this method is referred to as the method of "vanishing viscosity". If $H \in W_{\text {loc }}^{1, \infty}\left(\mathbf{R}^{N}\right)$ and $u_{0} \in W^{1, \infty}\left(\mathbf{R}^{N}\right)$, standard results and methods for quasilinear partial differential equations yield the existence and uniqueness of a solution $u^{\varepsilon}$ of (IVP) $)_{\varepsilon}$ in the class $\operatorname{BUC}\left(\mathbf{R}^{N} \times[0, T]\right) \cap C^{2,1}\left(\mathbf{R}^{N} \times(0, T)\right)$ (i.e., continuous second order spatial and first order time derivatives) for all $T<\infty$. Our main result is

THEOREM 5.1. Assume $H$ is locally Lipschitz continuous on $\mathbf{R}^{N}, u_{0}$ is bounded and Lipschitz continuous on $\mathbf{R}^{N}$ and $T>0$. Then, if $u^{\varepsilon}$ denotes the solution of $(\mathrm{IVP})_{\varepsilon}$ and $u$ denotes the viscosity solution of (IVP), we have

$$
\sup _{0 \leqslant t \leqslant T} \sup _{x \in \mathbf{R}^{N}}\left|u^{\varepsilon}(x, t)-u(x, t)\right| \leqslant c \sqrt{\varepsilon},
$$

where $c$ depends only on the Lipschitz constants of $u_{0}, H$ and $T$.

Proof. To simplify the presentation we will only consider the case when $H(0)=0$ and $u, u^{\varepsilon} \rightarrow 0$ as $|x| \rightarrow \infty$ uniformly in $t \in[0, T](T<\infty)$. The general case is easily obtained by (now) routine adaptations of this simpler case [1], [2].

Assume

$$
\boldsymbol{\sigma}=\sup _{0 \leqslant t \leqslant T} \sup _{x \in \mathbf{R}^{N}}\left(u(x, t)-u^{\varepsilon}(x, t)\right)>0,
$$

and let $\psi: \mathbf{R}^{N} \times \mathbf{R}^{+} \times \mathbf{R}^{N} \times \mathbf{R}^{+} \rightarrow \mathbf{R}$ be given by

$$
\begin{aligned}
\psi(x, t, y, s)= & u(x, t)-u^{\varepsilon}(y, s)-\frac{\sigma}{4 T}(t+s) \\
& +\left(5 M+\frac{\sigma}{2}\right) \beta_{\alpha}(x-y, t-s),
\end{aligned}
$$

where $M=\left\|u_{0}\right\|, \alpha>0$ and $\beta_{\alpha}, \beta$ are as in (4.7), (4.9), (4.21). (We now use $x, y$ to denote points in $\mathbf{R}^{N}$.) Just as in the previous case, we conclude that there is a point $\left(x_{0}, t_{0}, y_{0}, s_{0}\right)$ which maximizes $\psi$ over $\left(\mathbf{R}^{N} \times[0, T]\right)^{2}$. Moreover

$$
\psi\left(x_{0}, t_{0}, y_{0}, s_{0}\right) \geqslant 5 M+\sigma \text { and } \beta_{\alpha}\left(x_{0}-y_{0}, t_{0}-s_{0}\right) \geqslant 3 / 5 \text {, }
$$

so that

$$
\left|x_{0}-y_{0}\right|^{2}+\left(t_{0}-s_{0}\right)^{2} \leqslant \alpha^{2} / 2 \text {. }
$$


As before, we will consider the cases $t_{0}, s_{0}>0 ; t_{0}=0, s_{0}>0$; and $t_{0} \geqslant 0, s_{0}=0$ separately. Before doing so, we review a few properties of $u^{\varepsilon}$. We have the elementary estimates

$$
\left|u^{\varepsilon}\right| \leqslant M, \quad\left|D u^{\varepsilon}\right| \leqslant\left\|D u_{0}\right\|_{L^{\infty}\left(\mathbf{R}^{N}\right)} \quad \text { in } \mathbf{R}^{N} \times(0, \infty)
$$

(see, e.g., [2], [10]). Therefore $H\left(D u^{\varepsilon}\right)$ is bounded independently of $\varepsilon$. The following lemma will then allow us to estimate the modulus of continuity in time of $u^{\varepsilon}$ in the form

$$
\left|u^{\varepsilon}(x, t)-u^{\varepsilon}(x, s)\right| \leqslant K \sqrt{\varepsilon}|t-s|^{1 / 2}+K|t-s| .
$$

LEMma 5.2. Let $v \in C^{2,1}\left(\mathbf{R}^{N} \times(0, \infty)\right) \cap W^{1, \infty}\left(\mathbf{R}^{N} \times[0, \infty)\right)$ satisfy $\left|v_{t}-\varepsilon \Delta v\right| \leqslant$ $K_{0}$ in $\mathbf{R}^{N} \times(0, \infty)$. Then there is a constant $K$ depending only on $K_{0}$ and $\sup _{t>0}\|D v\|_{L^{\infty}\left(\mathbf{R}^{N}\right)}$ such that

$$
|v(x, t)-v(x, s)| \leqslant K\left(\sqrt{\varepsilon}|t-s|^{1 / 2}+|t-s|\right) \quad \text { for } x \in \mathbf{R}^{N}, t, s \geqslant 0 .
$$

Proof. Let $\rho \in C^{\infty}\left(\mathbf{R}^{N}\right)$ be a standard mollifier supported in the unit ball and satisfying $\int \rho(x) d x=1$. Put $v_{\alpha}=\rho_{\alpha} * v$. Clearly $\left|v_{\alpha t}-\varepsilon \Delta v_{\alpha}\right| \leqslant K_{0}$ in $\mathbf{R}^{N}$. Thus

$$
\begin{aligned}
\left\|v_{\alpha t}\right\|_{L^{\infty}\left(\mathbf{R}^{N}\right)} & \leqslant K_{0}+\varepsilon\left\|\Delta v_{\alpha}\right\|_{L^{\infty}\left(\mathbf{R}^{N}\right)} \\
& \leqslant K_{0}+\frac{c \varepsilon}{\alpha}\left\|D v_{\alpha}\right\|_{L^{\infty}\left(\mathbf{R}^{N}\right)} \leqslant K_{0}+\frac{c \varepsilon}{\alpha}\|D v\|_{L^{\infty}\left(\mathbf{R}^{N}\right)} \text { for } t \geqslant 0,
\end{aligned}
$$

where $c$ depends only on $\rho$. Therefore

$$
\begin{aligned}
|v(x, t)-v(x, s)| \leqslant & \left|v(x, t)-v_{\alpha}(x, t)\right|+\left|v(x, s)-v_{\alpha}(x, s)\right| \\
& +\left(K_{0}+\frac{K \varepsilon}{\alpha}\right)|t-s| \\
\leqslant & K(\alpha+|t-s|+(\varepsilon / \alpha)|t-s|),
\end{aligned}
$$

where $K$ denotes several constants with allowed dependencies. Setting $\alpha=$ $\varepsilon^{1 / 2}|t-s|^{1 / 2}$ yields the result.

We now turn to the cases $t_{0} \geqslant 0, s_{0}=0$, and $t_{0}=0, s_{0}>0$. Here only continuity considerations are involved, as before. If $t_{0} \geqslant 0$ and $s_{0}=0$, we have

$$
\begin{aligned}
5 M+\sigma & \leqslant \psi\left(x_{0}, t_{0}, y_{0}, 0\right) \\
& \leqslant 5 M+\sigma / 2+\left|u\left(y_{0}, t_{0}\right)-u\left(x_{0}, t_{0}\right)\right|+\left|u\left(x_{0}, 0\right)-u\left(x_{0}, t_{0}\right)\right| \\
& \leqslant 5 M+\sigma / 2+c\left(\left|x_{0}-y_{0}\right|+t_{0}\right) .
\end{aligned}
$$

Now, in a manner similar to (but simpler than) the 3rd case in Section 4, we can conclude that $\left|x_{0}-y_{0}\right|$ and $t_{0}$ are bounded by multiples of $\alpha^{2}$ and so $\sigma \leqslant c \alpha^{2}$ in this case. If $\alpha=\varepsilon^{1 / 4}$, we have the desired estimate. The case $t_{0}=s_{0}=0$ is subsumed under the one just treated. If $t_{0}=0$ and $s_{0}>0$, we have

$$
\begin{aligned}
u^{\varepsilon}\left(y_{0}, s_{0}\right)-( & \left.5 M+\frac{\sigma}{2}\right) \beta_{\alpha}\left(x_{0}-y_{0},-s_{0}\right)+\frac{\sigma}{4 T} s_{0} \\
& \leqslant u^{\varepsilon}\left(y_{0}, 0\right)-\left(5 M+\frac{\sigma}{2}\right) \beta_{\alpha}\left(x_{0}-y_{0}, 0\right) .
\end{aligned}
$$

From this and (5.5), (5.7) we deduce an estimate

$$
s_{0}^{2} / \alpha^{2} \leqslant K\left(\sqrt{\varepsilon} \sqrt{s_{0}}+s_{0}\right),
$$


where $K$ is independent of $\alpha$. This implies that $s_{0} \leqslant K\left(\varepsilon^{1 / 3} \alpha^{4 / 3}+\alpha^{2}\right)$. Now

$$
5 M+\sigma<\psi\left(x_{0}, 0, y_{0}, s_{0}\right) \leqslant 5 M+\sigma / 2+K \alpha^{2}+K \sqrt{\varepsilon} \sqrt{s_{0}}+K s_{0} .
$$

Using the previous estimate and letting $\alpha=\varepsilon^{1 / 4}$, we conclude that $\sigma \leqslant K \varepsilon^{1 / 2}$.

The final case, $s_{0}>0, t_{0}>0$, uses the equations satisfied by $u$ and $u^{\varepsilon}$. From the fact that $u$ is Lipschitz continuous in $x$ and $t$ we deduce that

$$
\left|D_{x} \beta_{\alpha}\left(x_{0}-y_{0}, t_{0}-s_{0}\right)\right|, \quad\left|D_{t} \beta_{\alpha}\left(x_{0}-y_{0}, t_{0}-s_{0}\right)\right|
$$

are bounded, where $D_{x}, D_{t}$ refer to the derivatives of $\beta_{\alpha}(x, t)$. This implies

$$
\left|x_{0}-y_{0}\right|,\left|t_{0}-s_{0}\right| \leqslant K \alpha^{2} \text {. }
$$

Using that $u$ is a viscosity solution, we have

$$
\begin{aligned}
\sigma / 2 T-(5 M+\sigma / 2)\left(D_{t} \beta_{\alpha}\right)\left(x_{0}-y_{0}, t_{0}-s_{0}\right) \\
+H\left(-(5 M+\sigma / 2)\left(D_{x} \beta_{\alpha}\right)\left(x_{0}-y_{0}, t_{0}-s_{0}\right)\right) \leqslant 0 .
\end{aligned}
$$

On the other hand, using $u^{\varepsilon} \in C^{2,1}$ and that $\left(y_{0}, s_{0}\right)$ minimizes $(y, s) \rightarrow$ $\psi\left(x_{0}, t_{0}, y, s\right)$ over $\mathbf{R}^{N} \times[0, T]$ we also have

$$
\begin{gathered}
u_{t}^{\varepsilon}\left(y_{0}, s_{0}\right)+\sigma / 2 T+(5 M+\sigma / 2)\left(D_{t} \beta_{\alpha}\right)\left(x_{0}-y_{0}, t_{0}-s_{0}\right) \leqslant 0, \\
D u^{\varepsilon}\left(y_{0}, s_{0}\right)+(5 M+\sigma / 2)\left(D_{x} \beta_{\alpha}\right)\left(x_{0}-y_{0}, t_{0}-s_{0}\right)=0,
\end{gathered}
$$

and

$$
\Delta u^{\varepsilon}\left(y_{0}, s_{0}\right)-(5 M+\sigma / 2)\left(\Delta \beta_{\alpha}\right)\left(x_{0}-y_{0}, t_{0}-s_{0}\right) \geqslant 0 .
$$

Now proceed by using $u_{t}^{\varepsilon}+H\left(D u^{\varepsilon}\right)-\varepsilon \Delta u^{\varepsilon}=0$, and (5.10), (5.12) to deduce that

$$
\begin{aligned}
& \varepsilon(5 M+\sigma / 2)\left(\Delta \beta_{\alpha}\right)\left(x_{0}-y_{0}, t_{0}-s_{0}\right) \\
& \quad-H\left(-(5 M+\sigma / 2)\left(D_{x} \beta_{\alpha}\right)\left(x_{0}-y_{0}, t_{0}-s_{0}\right)\right) \\
& \quad+\sigma / 2 T+(5 M+\sigma / 2)\left(D_{t} \beta_{\alpha}\right)\left(x_{0}-y_{0}, t_{0}-s_{0}\right) \leqslant 0 .
\end{aligned}
$$

Now, using (5.9), we conclude that

$$
\sigma / 2 T \leqslant \varepsilon(5 M+\sigma / 2)\left(\Delta \beta_{\alpha}\right)\left(x_{0}-y_{0}, t_{0}-s_{0}\right),
$$

and this yields $\sigma \leqslant K \varepsilon / \alpha^{2}$. Again, if $\alpha=\varepsilon^{1 / 4}$, we have the desired estimate.

Remark. By contrast with the analysis in Section 4, the Lipschitz continuity of $H$ was used only to assert that (IVP) $)_{\varepsilon}$ has a smooth solution. If $H$ is merely continuous, one still has $u_{t}^{\varepsilon}$ and $\Delta u^{\varepsilon}$ in $L_{\mathrm{loc}}\left(\mathbf{R}^{N} \times(0, \infty)\right)$ for $1 \leqslant p<\infty$, and the estimate (5.1) can still be proved.

Department of Mathematics

University of Wisconsin-Madison

Madison, Wisconsin 53706

Ceremade

Université Paris IX

Place de-Lattre-de-Tassigny

75775 Paris Cedex 16

France

1. M. G. Crandall, L. C. Evans \& P. L. Lions, Some Properties of Viscosity Solutions of Hamilton-Jacohi Equations, Math. Res. Center TSR \#2390, June 1982; Trans. Amer. Math. Soc. (To appear.)

2. M. G. Crandali. \& P. L. Lions, "Viscosity solutions of Hamilton-Jacobi equations," Trans. Amer. Math. Soc.. v. 277. 1983, pp. 1-42. 
3. M. G. Crandall \& P. L. Lions, "Conditions d'unicité pour les solutions généraliseés d'équations de Hamilton-Jacobi de premier ordre," C. R. Acad. Sci. Paris, v. 292, 1981, pp. 183-186.

4. M. G. Crandall \& A. Majda, "Monotone difference approximations for scalar conservation laws," Math. Comp., v. 34, 1980, pp. 1-21.

5. M. G. Crandall \& L. Tartar, "Some relations between nonexpansive and order preserving mappings," Proc. Amer. Math. Soc., v. 79, 1979, pp. 74-80.

6. L. C. Evans, "Some max-min methods for the Hamilton-Jacobi equation," Indiana Univ. Math. J., v. 33,1984 , pp. $31-50$.

7. W. H. Fleming, "Nonlinear partial differential equations-Probabilistic and game theoretic methods," Problems in Nonlinear Analysis, CIME, Edizioni Cremonese, Roma, 1971.

8. S. N. KRUŽKov, "The method of finite differences for a nonlinear equation of the first order with several independent variables," Ž. Vyčisl. Mat. i Mat. Fiz.,v. 6, 1966, pp. 884-894. (Russian)

9. N. N. KuZNETSOv, "On stable methods for solving non-linear first order partial differential equations in the class of discontinuous functions," Topics in Numerical Analysis III (J. J. H. Miller, Ed.), Academic Press, New York, 1977, pp. 183-197.

10. P. L. Lions, Generalized Solutions of Hamilton-Jacobi Equations, Pitman Lecture Notes, London, 1982.

11. P. E. Souganidis, “Approximation schemes for viscosity solutions of Hamilton-Jacobi equations," J. Differential Equations. (To appear.) 